\title{
Podoplanin: a Prognostic Marker and a Potential Target for Therapy in Bladder Cancer
}

\author{
TIBERIU ADELMANN ${ }^{1}$, NELA PUSA GAJE ${ }^{1,2}$, LAURA CRISTINA RUSU ${ }^{3 *}$ \\ 1 "Victor Babes" University of Medicine and Pharmacy, Department of Microscopic Morphology/Histology, 2 Eftimie Murgu \\ Sq, 300041, Timisoara, Romania \\ 2"Victor Babes" University of Medicine and Pharmacy, Angiogenesis Research Center Timisoara, 2 Eftimie Murgu Sq, \\ 300041, Timisoara, Romania \\ 3"Victor Babes" University of Medicine and Pharmacy, Department of Dentistry/Oral Pathology, 2 Eftimie Murgu Sq, 300041, \\ Timisoara, Romania
}

Invasive bladder cancer is a frequent neoplastic diseases, and despite progresses made in early diagnosis and surgical procedures, the outcome of patients is characterized by high rate of mortality. This is mainly due to the lack of response to chemotherapy and radiotherapy, and other new resources are limited. In the present work we analyzed 50 consecutive cases of invasive bladder cancer treated by open surgery. Specimens were processed using standard histological procedures, including establishment of the histological diagnosis and grading. Additional slides were stained for podoplanin expression, clone D2-40 to investigate the structure and number of lymphatic vessels. We found lymphatic vessels in both intra- and peritumor areas, showing significant differences in morphology and number. Lymphatic invasion by tumor cells was higher in peritumor than in intratumor vessels. Lymphatic microvessel density correlated with stage and grade of the tumor. Of interest is the expression of podoplanin by tumor cells of urothelial carcinoma in about $14 \%$ of the cases, with strong correlation with grading. Based on our results, expression of podoplanin in invasive bladder cancer might indicate three potential targets: lymphatics, myofibroblasts, and tumor cells in selected cases.

Keywords: bladder cancer, immunohistochemistry, podoplanin, therapeutic target

Investigation of the lymphatic system although initiated more than one hundred years ago, is a relatively new acquisition in biology and medicine. This is mainly due to the lack of specific markers of the lymphatic vessels endothelium (LECs). First specific markers were introduced only two decades ago, LYVE-1 [Banerjee et al, 1999], Prox-1 [Wigle and Oliver, 1999], and podoplanin [Breiteneder-Geleff et al, 1999]. Although not highly specific, these markers discriminate between blood and lymphatic vessels in both normal and pathological tissues. After early publications on this topic, there were published many data related to the development of lymphatic vessels network in normal and pathological conditions, and particularly, in malignant tumors. Immunohistochemistry is very useful to investigate the structure, number and particular aspects of lymphatic vessels, and these characters were designated as lymphangiogenesis.

Lymphangiogenesis is the process of formation of new lymphatic vessels from postcapillary venules, preexisting lymphatics, and/or directly from committed mesenchymal cells. Although of great importance for pathology, the origin of newly formed lymphatics in normal and pathological conditions is not yet completely clarified [Shayan et al, 2006, He et al, 2004]. Lymphangiogenesis is frequently associated with the natural evolution and spreading of a large variety of carcinoma. After the characterization of specific markers of LECs, there were accumulated data that support their proliferative potential in normal tissues, but also in tumor specimens [Wilting et al, 2003; Jeltsch et al, 2003]. In an effort to better characterize the molecular profile of LECs, there were identified other specific markers, like receptor 3 of vascular endothelial growth factor (VEGFR3) or desmoplakin. Investigating how specific these markers are, it was found that LYVE-1 stained endothelial cells of hepatic sinusoids, activated macrophages, and about $10 \%$ of blood vessels from the tumor area.

*email:: laura.rusu@umft.ro 
Based on the data accumulated in last two decades it was appreciated that podoplanin (Aggrus/T1alpha/ PA2.26/gp36/M2A) shows the highest specificity and may be used in paraffin sections and identified by immunohistochemistry. An accurate technique to demonstrate lymphatic endothelium is crucial to evaluate lymphatic microvessel density (LMVD), and on the other hand, to evaluate the prognostic role of podoplanin expression in some tumor cells. Podoplanin mRNA has been identified for the first time in murine osteoblasts [Nose et al, 1990]. The first report of podoplanin expression in LECs and other non-endothelial cells belong to Wetterwald et al [1996], which called this antigen E11, and then podoplanin, based on the low level of expression in normal kidney podocytes.

The difference between blood and lymphatic vessels has been always a problem in morphological studies, because the decision implies the subjectivism of the examiner. Podoplanin is very useful to discriminate between vessels, and the antibody D2-40 that recognize the formalin-insensitive epitope of podoplanin is the most largely used [Marks et al, 2000]. The status of intratumoral and peritumoral lymphatic vessels is particularly important in developing of lymph node metastases associated to bladder cancer. However, lympho-vascular invasion is well known as predictive for lymph node metastases. Despite these data, studies on the lymphatic vessels and lymphangiogenesis in invasive bladder cancer are relatively rare, and results are controversial. On the other hand, LMVD seems to be an individual prognostic marker in various tumors, like carcinoma of the uterine cervix, head and neck squamous cell carcinoma, ovarian carcinoma and malignant melanoma (Raica et al, 2013; Raica et al, 2015; Cobec et al, 2016).

Lymphangiogenesis is a dynamic process, stimulated by growth factors, and from these, the most efficient seems to be vascular endothelial growth factor-C (VEGF-C). VEGF-C could be secreted by tumor cells themselves, but also by some cells of the tumor microenvironment. LECs proliferation and migration is induced by the binding of VEGF-C to its cognate receptor, VEGFR-3. Activation of VEGFR-3 stimulates formation of new lymphatics in both intratumoral and peritumoral areas. Most of the publication in the field demonstrate the relationship between overexpression of VEGF-C/VEGFR-3 and highly aggressive tumor, but until now the type of bladder cancer that might benefit from inhibitory therapy was not defined.

Opposite to angiogenesis, currently there are not known approved inhibitory drugs of lymphangiogenesis for clinical application. Only experimental studies that used anti-podoplanin monoclonal antibodies (NZ-1) and antiVEGF-C with non-convincing data are available. For all these reasons, we believe that characterization of potential therapeutic targets identified with anti-podoplanin is important in invasive bladder cancer, as conventional adjuvant therapy fails in most of the cases.

\section{Experimental part}

Material and methods

Patients

There were included in the study 50 consecutive patients with invasive tumors of the urinary bladder, T2-T4, ranged between 56 and 72 years. Diagnosis was based on clinical, imagistic, endoscopic, and pathological criteria, according to standard procedures. All patients were treated by radical cystectomy followed by construction of low pressure reservoir or urinary diversion, depending on the location and local extension of the tumor. Biopsies were taken from the primary tumor and from the border between tumor and apparently normal tissue.

\section{Primary processing}

Biopsies were processed according the standard histological technique. Briefly, they were washed in buffer saline and fixed in buffer formalin $p \mathrm{H} 7.2$ for 72 hours. After dehydration and clarification, they were embedded in paraffin and from each block there were performed $3 \mu \mathrm{m}$ sections. The full procedure was automatic, using Thermo Shandon devices. As conventional staining, we used hematoxylin-eosin for the diagnosis and estimation of the grading. Additional sections were performed for the immunohistochemical evaluation.

Immunohistochemistry has been performed using Leica Bond-Max (Leica Biosystems, Newcastle upon Tyne, UK). Antigen retrieval was performed with Bond Epitope Retrieval Solution 2 for 20 de minutes (Leica Biosystems, Newcastle Ltd). Endogenous peroxydase was blocked with hydrogen peroxide 3\%, for 5 minutes. Sections were then treated with the primary antibody, clone D2-40 (Santa Cruz, ready-to-use) and the reaction was conducted with Bond Polymer Refine Detection System (8 minutes). Finnaly, the product of reaction was 
visualized in brown with 3, 3' diamino benzidine dihydrochloride (10 minutes), and nuclei were stained with hematoxylin.

\section{Microscopic evaluation and image analysis}

Stained sections were analyzed with Zeiss Axiocam 506 (Jena, Germany), including the digital system of image processing and soft dedicated to microscopic image analysis. LMVD was calculated based on the standard protocol proposed by Weidner et al (1994). Double immunostaining has been evaluated using the semi-automated method proposed by Suciu et al (2014).

Statistical methods were applied to evaluate the relationships between LMVD and/or podoplanin expression and clinical and pathological conventional parameters. Student test and Chi square were used in this study and $\mathrm{p}<0.5$ was considered statistically significant.

\section{Results and discussions}

In the first step we evaluated the sensitivity and specificity of D2-40 antibody in relation with LECs. In all cases, the final product of reaction was restricted to LECs, but not to the endothelium of blood vessels. The pattern of the positive reaction was cytoplasmic, diffuse, and strong in all lymphatic endothelial cells. Lymphatic vessels were identified in the intratumoral and peritumoral areas in all cases included in the study. On one hand, the immunoreaction for podoplanin was highly sensitive with the lymphatic endothelium, because it constantly does not stain blood vessels endothelium. The specificity of reaction is not absolute, because other non-endothelial cell may show positive reaction, like, basal cells of the urothelium (fig.1a) and nerve fibers (Schwann cells) (fig. 1b). We used these structures as internal positive control of the reaction for podoplanin.

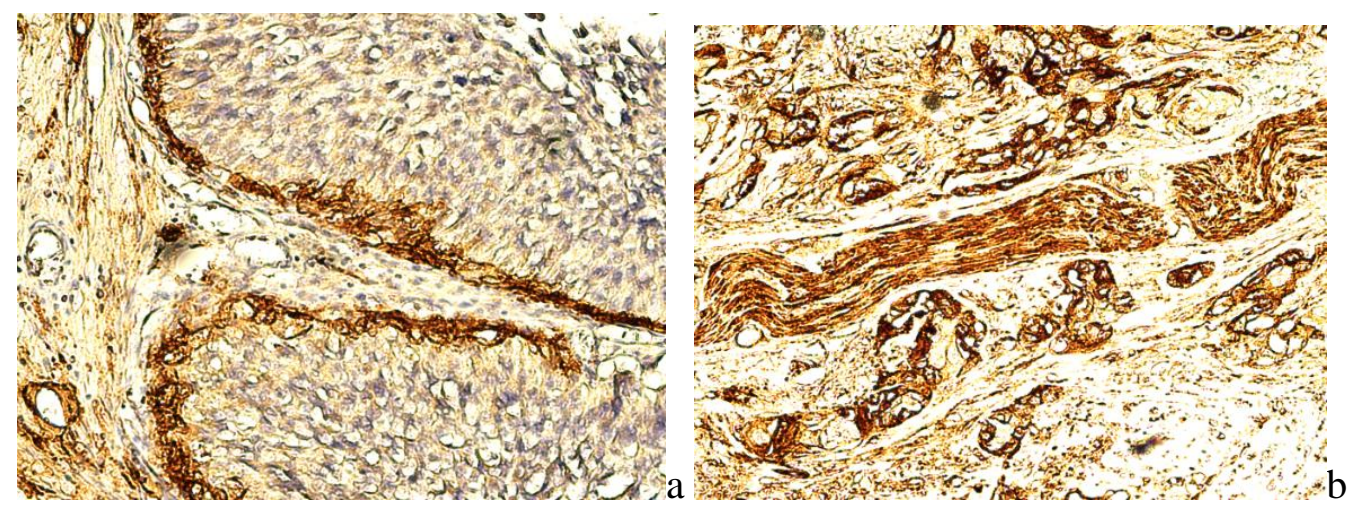

Fig. 1. Basal cell layer of the urothelium (a). Nerve fiber surrounded by small nests of tumor cells (b). Reaction for podoplanin, $\mathrm{x} 400$.

Lymphatic vessels were found in all cases included in the study in both intratumoral and peritumoral areas. Intratumoral lymphatic vessels were usually small, with narrow and irregular lumen (fig.2a). We identified tumor cells in intratumoral vessels in 3 cases. Peritumoral lymphatic vessels were larger, with large lumen, frequently located close to blood vessels (fig.2b). Lymphovascular invasion was noticed in 12 from 50 (24\%) cases on slides stained for podoplanin (fig.3c) in comparison with the conventional stained slides (7 from 50, 14\%). 


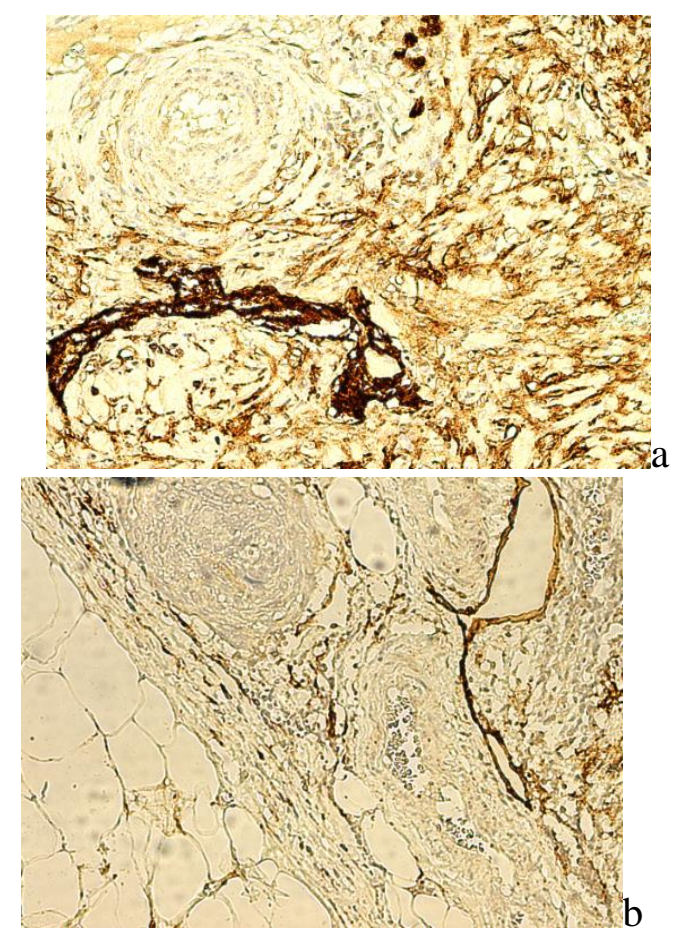

Fig. 2. Intratumoral lymphatic vessel (a). Peritumoral lymphatic vessel (b). Lymphovascular invasion (c). Immunoreaction for podoplanin

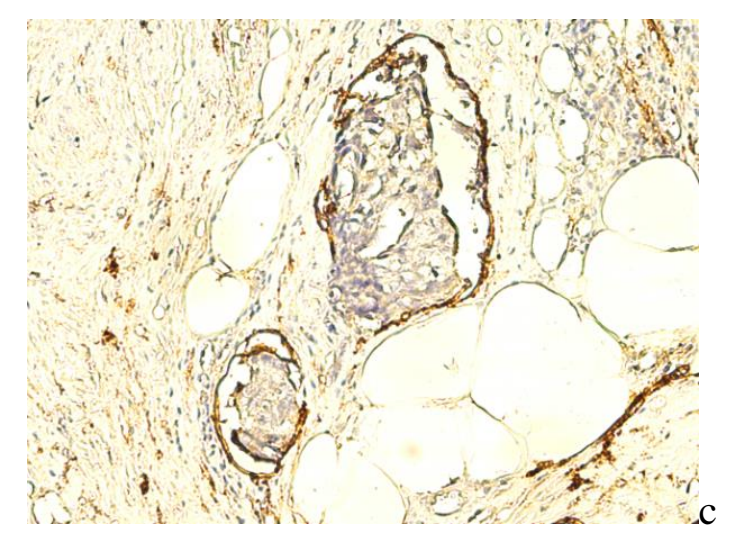

The number of lymphatic vessels is higher in the peritumoral area in 48 from 50 cases. To count lymphatic microvessel we have chosen three fields from each slide with maximum density of positive and intensely stained vascular structures. We found a positive correlation between values of LMVD (see Table 1) and grading $(\mathrm{p}<0.0023)$. Counting was particularly difficult in the tumor microenvironment rich in myofibroblasts, and in isolated cases $(n=3)$ were intratumoral lymphatic vessels were arranged similar to glomeruloid bodies.

Table 1 LYMPHATIC MICROVESSEL DENSITY IN INVASIVE BLADDER CANCER

\begin{tabular}{|c|c|c|}
\hline Histological type & Intratumoral & Peritumoral \\
\hline Urothelial carcinoma $(\mathrm{n}=47)$ & 16.4 & 22.4 \\
\hline Squamous cell carcinoma $(\mathrm{n}=2)$ & 11.2 & 12.5 \\
\hline Adenocarcinoma $(\mathrm{n}=1)$ & 6.5 & 8.7 \\
\hline
\end{tabular}

We found expression of podoplanin in tumor cell in 8 from 47 invasive urothelial carcinoma (17.2\%). The final product of reaction showed a cytoplasmic, diffuse and homogeneous pattern, stronger at the front of proliferation (fig.3a) or heterogeneous (fig.3b). We noticed a strong correlation between the expression of podoplanin by tumor cells and grading (all 8 cases were G3). Expression of podoplanin by tumor cells could be an early acquisition during carcinogenesis because we found positive reaction in associated dysplasia close to the 
primary tumor. Squamous cell carcinoma, adenocarcinoma, and sarcomatoid carcinoma were consistently negative.

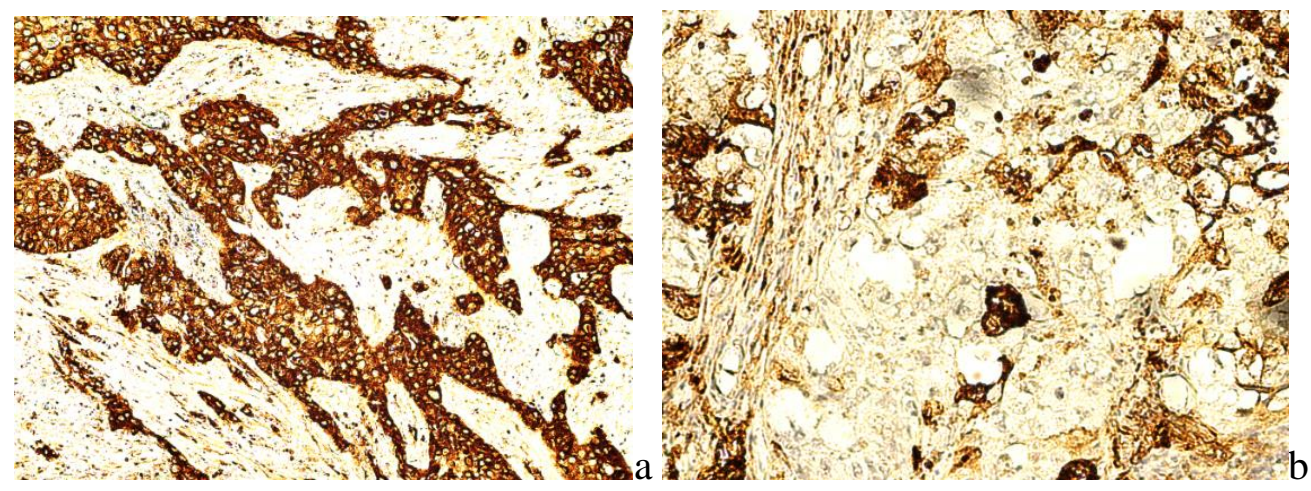

Fig.3. Expression of podoplanin by tumor cells in invasive bladder carcinoma. Homogeneous model of positive reaction (a). Heterogeneous model (b). Magnification x200.

In 43 from 50 cases (86\%) in the tumor microenvironment were stained a large number of myofibroblasts located close to the cords and islands of tumor cells and even in the lamina propria of dysplastic urothelium and carcinoma in situ. Usually, myofibroblasts were spindle shaped, with thick and branched cytoplasmic processes (fig.4). The myofibroblastic reaction seems to follow neoplastic cells as it was not found in any cases in noninvaded bladder wall.

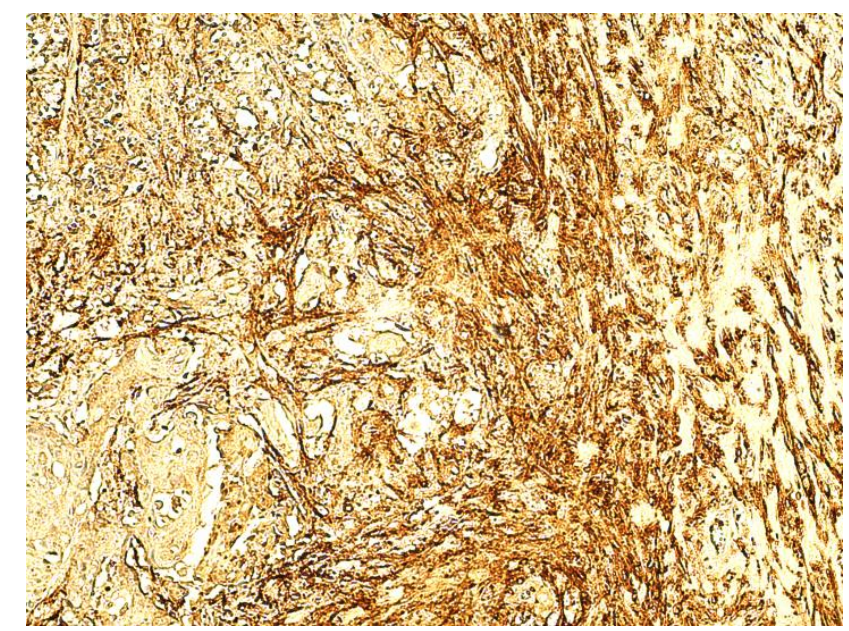

Fig. 4. Abundant myofibroblast reaction in the tumor microenvironment. Immunoreaction with anti-podoplanin, x200.

Podoplanin has been detected on the surface of rat podocytes, and it is a $38 \mathrm{kDa}$ membrane protein, related to the flattening of pedicels in puromicyn-induced nephrosis [Breiteneder-Geleff et al, 1997]. This animal model has been performed to reproduce at least in part, minimal changes human nephropathy, associated with a significant decrease in the expression of podoplanin. Based on these observations, it is believed that podoplanin plays an important role in maintaining of the unique shape of podocytes [Matsui et al, 1998].

In the present work we have shown that podoplanin is highly sensitive for LECs, but it has not absolute specificity. Investigating invasive bladder cancer, we observed a constant and strong reaction in myofibroblasts from the tumor microenvironment, and in tumor cells from $17.2 \%$ of the cases. On the other hand, by double immunohistochemistry lymphatic vessels can be differentiated from blood vessels. Using a method based on D240 and CD34, it has been shown that the final product of reaction is restricted to LECs, and does not stain blood vessels endothelium in both normal and pathological conditions [Xuan et al, 2005]. Therefore, a potential target for therapy in patients with invasive bladder cancer is represented by LECs. Currently, there are not available anti-LECs drugs that discriminate between normal and tumor-associated lymphatic vasculature.

PDPN gene is located on chromosome 1p36.21, is a functional gene with $34.2 \mathrm{~kb}$ and 8 exons, which controls podoplanin synthesis. The synthesized protein is subcellular located at membrane level. Two species of mRNA 
were identified by Northern blotting that most probably results from alternative segmentation [Kriehuber et al, 2001]. Podoplanin was originally generated during studies on platelet aggregation and it has been shown that 8F11 monoclonal antibody inhibits aggregation in vitro and metastasis of colorectal carcinoma in vivo in mice [Kato et al, 2003]. Podoplanin-induced aggregation of platelets takes place even in the absence of other components of the plasma [Kaneko et al, 2006]. Podoplanin belongs to the type 1 transmembrane sialomucins, consists of 162 amino acids, with an intracellular cytoplasmic short tail which binds proteinkinase $\mathrm{C}$ and phosphorylates cAMP, and an extracellular domain rich in serin and threonine. A specific endogenous receptor for podoplanin, a C-type lectin (CLEC-2), was identified on blood platelets [Suzuki-Inoue et al, 2007]. Blocking this receptor leads to the inhibition of podoplanin0induced platelet aggregation [Kato et al, 2007]. This mechanism explain in part the expression of podoplanin in a large variety of normal and tumor cells, and on the other hand, its involvement in tumor progression [Suzuki-Inoue et al, 2007]. We report here for the first time the immunohistochemical expression of podoplanin in tumor cells of the invasive bladder cancer. Notably, we found a strong correlation between this expression and grading. Therefore, a humanized monoclonal anti-podoplanin antibody might target not only LECs, but also tumor cells in some cases.

Podoplanin is involved in the formation of lymphatic vessels, and it is a constitutional marker of LECs, but the mechanism by which it acts in these conditions is not yet understood [Schacht et al, 2003]. Podoplanindeficient mouse is characterized by perinatal lethality induced by major lung abnormalities and respiratory failure. However, podoplanin expression has been reported in alveolar cells type 1. Validation of the role of podoplanin in the formation of lymphatics is supported by experimental data with VEGF-C that induces formation of vascular structures positive for both podoplanin and Prox-1 [Kreuger et al, 2006]. Podoplanin expression is regulated by Prox 1 gene, a marker that controls development of lymphatic progenitors from embryonal veins [Kato et al 2006]. In embryonal tissues the intensity of Prox 1 expression is moderate or strong, and the expression of podoplanin remains strong in the postnatal life [Petrova et al, 2002; Schacht et al, 2003]. All these data support the specificity of podoplanin/D2-40 for the lymphatic endothelium, including invasive tumors of the urinary bladder. Podoplanin is more specific than LYVE-1, which was expressed by only a subset of podoplanin-positive cells [Makinen et al, 2001; Ji et al, 2007]. This is why is the current study we used exclusively D2-40 to detect lymphatic vessels, and its formalin-insensitive epitope.

In the present study we have noticed the expression of podoplanin in a subset of high grade invasive tumors of the bladder. We described some different models of distribution of the final product of reaction, and the heterogeneous pattern was the most frequent. Expression of podoplanin in tumor cells has been reported before in a variety of cancers, like, mammary, gastric, thymus carcinoma or neuroblastoma (Raica et al, 2011; Raica et al, 2008; Ribatti et al, 2010; Raica et al, 2010). If the pattern of reaction is heterogeneous, the majority of podoplanin-tumor cells are located at the front of proliferation (Raica et al, 2008). To the best of our knowledge, it is the first report on the expression of podoplanin in malignant cells of invasive urothelial carcinoma. The correlation with grade of the tumor signals out the highly aggressive tumor and high capacity of local invasion.

Based on our results, the evaluation of podoplanin expression in invasive bladder tumors is of high interest in diagnosis, prognosis, and detection of potential therapeutic targets. A humanized anti-podoplanin antibody could act on a triple target, namely lymphatic endothelium, myofibroblasts of the tumor microenvironment, and in some selected cases, directly on tumor cells.

\section{Conclusions}

Our observations reveal the presence of intra- and peritumoral lymphatic vessels in 50 cases of invasive bladder cancers. Lymphovascular invasion has been detected in both but with higher incidence in peritumoral vessels. Lymphatic microvessel density correlates with stage and grade of the tumor. Podoplanin is expressed in tumor cells by $17.02 \%$ of urothelial carcinoma, and becomes an attractive therapeutic target. Our data support the routine detection of podoplanin expression in urinary bladder tumors in order to detect LECs, myofibroblasts and positive tumor cells.

\section{References}


1BANERJEE S, NI J, WANG SX, CLASPER S, SU J, TAMMI R, JONES M, JACKSON DG - LYVE-1, a new homologue of the CD44 glycoprotein, is a lymph-specific receptor for hyaluronan. J Cell Biol, 1999, 144: 789-801.

2.WIGLE JT, OLIVER G - Prox1 function is required for the development of the murine lymphatic system. Cell, 1999, 98: 769-778.

3.BREITENEDER-GELEFF S, MATSUI K, SOLEIMAN A ET AL - Podoplanin, novel $43 \mathrm{kDa}$ membrane protein of glomerular epithelial cells, is down-regulated in puromycin nephrosis. Am J Pathol, 1997, 151: 1141-1152.

3.MARKS A, SUTHERLAND DR, BAILEY D ET AL - Characterization and distribution of an oncofetal antigen (M2A antigen) expressed on testicular germ cell tumours. Br J Cancer, 2000, 80: 569-578.

5.WETTERWALD A, HOFSTETTER W, CECCHINI MG ET AL - Characterization and cloning of the E11 antigen, a marker expressed by the rat osteoblasts and osteocytes. Bone, 1996, 18:125-132.

6.NOSE K, SAITO H, KUROKI T - Isolation of a gene sequence induced later by tumor-promoting 12-O-tetradecanoylphorbol-13-acetate in mouse osteoblastic cells (MC3T3-E1) and expressed constitutively in ras-transformed cells. Cell Growth Differ, 1990, 1: 511-518.

7.WILTING J, TOMAREV SI, CHRIST B, SCHWEIGERER L - Lymphangioblasts in embryonic lymphangiogenesis. Lymphat Res Biol, 2003, 1: $33-40$.

8.JELTSCH M, TAMMELA T, ALITALO K, WILTING J - Genesis and pathogenesis of lymphatic vessels. Cell Tissue Res, 2003 , $314:$ 69-84.

9.SHAYAN R, ACHEN MG, STACKER SA - Lymphatic vessels in cancer metastasis: bridging the gap. Carcinogenesis, 2006, 27: 1729-1738.

10.HE Y, RAJANTIE I, ILMONEN M ET AL - Preexisting lymphatic endothelium but not endothelial progenitor cells are essential for tumor lymphangiogenesis and lymphatic metastasis. Cancer Res, 2004, 64: 3737-3740.

11.RAICA M, CIMPEAN AM, POPOVICI RA, BALICA AR, VLADAU M, GAJE PN. Mast cells stimulate lymphangiogenesis in the gingiva of patients with periodontal disease. In Vivo. 2015; 29(1):29-34.

12.COBEC IM, SAS I, PIRTEA L, CIMPEAN AM, MOATAR AE, CEAUȘU RA, RAICA M. Podoplanin as Key Player of Tumor Progression and Lymph Vessel Proliferation in Ovarian Cancer. Anticancer Res. 2016;36(10):5265-5272.

13.RAICA M, CIMPEAN AM, CEAUSU R, RIBATTI D, GAJE P. Interplay between mast cells and lymphatic vessels in different molecular types of breast cancer. Anticancer Res. 2013;33(3):957-63.

14.RAICA M, CIMPEAN AM, CEAUSU R, RIBATTI D. Lymphatic microvessel density, VEGF-C, and VEGFR-3 expression in different molecular types of breast cancer. Anticancer Res. 2011; 31(5):1757-64.

15.RAICA M, RIBATTI D, MOGOANTA L, CIMPEAN AM, IOANOVICI S. Podoplanin expression in advanced-stage gastric carcinoma and prognostic value of lymphatic microvessel density. Neoplasma. 2008;55(5):455-60.

16.RIBATTI D, NICO B, CIMPEAN AM, RAICA M. Podoplanin and LYVE-1 expression in lymphatic vessels of human neuroblastoma. J Neurooncol. 2010;100(1):151-152.

17.RAICA M, CIMPEAN AM, RIBATTI D. The role of podoplanin in tumor progression and metastasis. Anticancer Res. 2008;28(5B):2997-3006.

18.MATSUI K, BREITENEDER GELEFF S, KERJASCHKI D - Epitope specific antibodies to the $43 \mathrm{kD}$ glomerular membrane protein podoplanin cause proteinuria and rapid flattening of podocytes. J Am Soc Nephrol, 1998, 9: 2013-1026.

19.XUAN M, FANG YR, WATO M ET AL - Immunohistochemical co-localization of lymphatics and blood vessels in oral squamous cell carcinomas. J Oral Pathol Med, 2005, 34: 334-339.

20.MAKINEN T, JUSSILA L, VEIKKOLA T, KARPANEN T, KETTUNEN MI, PULKKANEN KJ, KAUPPINEN R, JACKSON DG, KUBO H, NISHIKAWA S, YLA-HERTTUALA S, ALITALO K. Inhibition of lymphangiogenesis with resulting lymphedema in transgenic mice expressing soluble VEGF receptor-3. Nature Med, 2001, 7: 199-205.

21.HE Y, KOZAKI K, KARPANEN T, KOSHIKAWA K, YLA-HERTTUALA S, TAKAHASHI T, ALITALO K - Supression of tumor lymphangiogenesis and lymph node metastasis by blocking vascular endothelial growth factor receptor 3 signalling. J Natl Cancer Inst, 2002, 94: 819825 .

22.KANEKO MK, KATO Y, KUNITA A ET AL - Conservation of a platelet activating domain of Aggrus/podoplanin as a platelet aggregationinducing factor. Gene, 2006, 378: 52-57.

23.SUZUKI-INOUE K, KATO Y, INOUE O ET AL - Involvement of the snake toxin receptor CLEC-2 in podoplanin-mediated platelet activation by cancer cells. J Biol Chem, 2007, 282: 25993-26001.

24.KREUGER J, NILSSON I, KERJASCHKI D ET AL - Early lymph vessel development from embryonic stem cells. Arterioscler Thromb Vasc Biol, 2006, 26: 1073-1078.

25.KATO Y, KANEKO MK, KUNO A ET AL - Inhibition of tumor cell-induced platelet aggregation using a novel anti-podoplanin antibody reacting with its platelet-aggregation-stimulating domain. Biochem Biophys Res Commun, 2006, 349: 1301-1307.

26.PETROVA TV, MAKINEN T, MAKELA TP ET AL - Lymphatic endothelial reprogramming of vascular endothelial cells by the Prox-1 homebox transcription factor. EMBO J. 2002, 21: 4593-4599.

27.JI RC, ESHITA Y, KATO S - Investigation of intratumoural and peritumoral lymphatics expressed by podoplanin and LYVE-1 in the hybridomainduced tumours. Int J Exp Path, 2007, 88: 257-270.

28.SCHACHT V, RAMIREZ MI, HONG YK ET AL - T1 $\alpha$ /podoplanin deficiency disrupts normal lymphatic vasculature formation and causes lymphedema. EMBO J, 2003, 22: 3546-3556.

29.KRIEHUBER E, BREITENEDER-GELEFF S, GROEGER M, SOLEIMAN A, SCHOPPMAN SF, STINGL G, KERJASSCKI D, MAURER D Isolation and characterization of dermal lymphatic and blood endothelial cells reveal stable and functionally specialized cell lineages. J Exp Med, 2001, 194: 797-808.

Manuscript received: 22.11 .2019 